\title{
Uniformly Controllable Schemes for the Wave Equation on the Unit Square
}

\author{
M. $\mathrm{ASCH}^{*}$ and A. MÜNCH ${ }^{\dagger}$ \\ Communicated by R. Glowinski
}

${ }^{*}$ LAMFA, UMR CNRS 6140, Université de Picardie Jules Verne, 33 rue St. Leu, 80039 Amiens, France, (mark. asch@u-picardie.fr).

${ }^{\dagger}$ Laboratoire de Mathématiques de Besançon, UMR CNRS 6623, Université de Franche-Comte, 16 route de Gray 25030 Besançon cedex, France, (arnaud.munch@univ-fcomte.fr). 


\begin{abstract}
The paper deals with the numerical approximation of the HUM control of the 2-D wave equation. Most of the discrete models obtained with classical finite difference or finite element methods do not produce convergent sequences of discrete controls, as the mesh size $h$ and the time step $\Delta t$ go to zero. We introduce a family of full-discrete schemes, nondispersive, stable under the condition $\Delta t \leq h / \sqrt{2}$ and uniformly controllable with respect to $h$ and $\Delta t$. These implicit schemes differ from the usual explicit one (obtained with leapfrog time approximation and five point spatial approximations) by the addition of terms proportional to $h^{2}$ and $\Delta t^{2}$. Numerical experiments for nonsmooth initial conditions on the unit square using a conjugate gradient algorithm indicate the excellent performance of the schemes.
\end{abstract}

Keywords : Uniformly exact controllability, 2D wave equation, Numerical approximation. 


\section{Introduction}

Let $\Omega=(0,1)^{2}$ and $\Gamma_{0}\left(x_{0}\right)=\left\{x \in \partial \Omega,\left(x-x_{0}\right) \cdot \vec{\nu} \geq 0\right\}$ for all $x_{0} \in \mathbb{R}^{2}$, where $\vec{\nu}$ is the outward normal vector. In the context of exact controllability, the following result is well-known [1]: given a time $T>T^{\star}(\Omega)$ large enough and $\left(y^{0}, y^{1}\right) \in L^{2}(\Omega) \times H^{-1}(\Omega)$, there exists a control $v \in L^{2}\left(\Gamma_{0} \times(0, T)\right)$ such that the solution $y$ of

$$
\begin{aligned}
y^{\prime \prime}-\Delta y=0, & \text { in } \Omega \times(0, T), \\
y=v \mathcal{X}_{\Gamma_{0}}, & \text { on } \partial \Omega \times(0, T), \\
\left(y(\cdot, 0), y^{\prime}(\cdot, 0)\right)=\left(y^{0}, y^{1}\right), & \text { in } \Omega,
\end{aligned}
$$

satisfies $\left(y(T, \cdot), y^{\prime}(T, \cdot)\right)=(0,0)$ in $\Omega$, where $\mathcal{X}_{\Gamma_{0}} \in L^{\infty}(\partial \Omega ;\{0,1\})$ denotes the characteristic function of $\Gamma_{0}$. This controllability problem has been studied and solved some decades ago. We mention the most successful moments theory [2] and more recently the Hilbert uniqueness method (HUM). The exact controllability is related to the observability inequality for the homogeneous solution $w$ with initial condition $\left(w^{0}, w^{1}\right) \in$ $H_{0}^{1}(\Omega) \times L^{2}(\Omega)$,

$$
\left\|w^{0}, w^{1}\right\|_{H_{0}^{1}(\Omega) \times L^{2}(\Omega)}^{2} \leq C_{T} \int_{0}^{T} \int_{\Gamma_{0}} w(x, t)^{2} d \sigma d t .
$$

We address the numerical approximation of this problem, known to be extremely delicate since the pioneering work of Glowinski-Li-Lions [3]. Using the HUM approach and usual finite difference schemes, say $\left(\mathcal{S}_{h, \Delta t}\right)$ consistent with $(\mathcal{S})$ and stable under the condition $\Delta t \leq h / \sqrt{2}$, these authors have observed the nonconvergence of the associate discrete controls $\left\{v_{h, \Delta t}\right\}_{(h, \Delta t)}$ toward the control $v$ when the mesh size $h$ and the time step $\Delta t$ go to zero. This by now well-known phenomenon (see [4] for a review) is due to the fact that $\left(\mathcal{S}_{h, \Delta t}\right)$ generates spurious high-frequency oscillations that do not exist at the continuous level. Moreover, the interaction of waves with the grid produces a dispersion phenomenon and the velocity of propagation of the high frequency numerical waves may converge to zero with the mesh size. Therefore, for these frequencies, the uniform controllability properties of the discrete model may disappear for a fixed time $T>T^{\star}$ independent of $h$ and $\Delta t$.

Several techniques have been proposed as cures of the spurious oscillations: Tychonoff regularization procedure in [3], multigrid strategy in [5,6], mixed finite-element methods in [7], filtering of the high frequency modes in [8]. From a theoretical viewpoint, this problem has been analyzed at the semidiscrete level (discretization in space) highlighting the role of the semidiscrete spectrum of $\left(\mathcal{S}_{h}\right)$. In [9], a semidiscrete finite element scheme for the 2-D wave equation, uniformly controllable with respect to $h$, was introduced. A discrete multiplier technique permits one to obtain a uniform semidiscrete observability inequality and then to prove the convergence of the semidiscrete sequence $\left\{v_{h}\right\}_{(h>0)}$. A step forward was recently made in [10] where the second author introduces and studies a full-discrete 1D implicit scheme. This scheme is a modification of the usual scheme $\left(\mathcal{S}_{h, \Delta t}\right)$ by the addition of some viscosity terms of order $\left(h^{2}-\Delta t^{2}\right)$, in the spirit of [11]. The proof of the uniform controllability under the condition $\Delta t \leq h \sqrt{T / 2}$ is obtained by means of a discrete Ingham inequality.

In the numerical experiments, we observe that the additional approximation in time (usually a centered leapfrog scheme) does not perturb the property of any uniformly controllable, semidiscrete scheme. As a consequence, the developments at the semidiscrete level permit one to design several full-discrete schemes leading to convergent sequences of controls $\left\{v_{h, \Delta t}\right\}_{(h, \Delta t>0)}$. However a challenging task still remains: how to obtain not only a uniformly controllable but also an efficient scheme in terms of execution time and rate of convergence. This difficulty is explained by the balance between the stability property and the group velocity property. The uniform controllability may be obtained, roughly, by increasing the dispersion (for high frequency components). However, this has the effect of deteriorating dramatically the stability condition. Thus, the semidiscrete scheme that we have recently introduced and thoroughly analyzed in [9] is uniformly 
controllable and has a group velocity, for high frequencies, of order of $h^{-3}$. However, at the full-discrete level, the corresponding scheme is stable under the condition $\Delta t=O\left(h^{3}\right)$.

In this paper, we obtain and analyze a uniformly controllable, fully-discrete scheme endowed with a good stability condition, typically $\Delta t=O(h)$. The method used (introduced in [10]) is constructive; it consists of considering a parameterized family of schemes, consistent with $(\mathcal{S})$, and then selecting, via a spectral analysis, the parameters leading to optimal uniform controllability and stability properties. This will lead us to a new scheme that is very efficient, implicit, very slightly dispersive, uniformly controllable and stable under the condition $\Delta t \leq h / \sqrt{2}$. This is done firstly at the semidiscrete level (Section 2) then at the fully discrete one (Section 3). Section 4 presents numerical experiments for a nonsmooth initial condition.

\section{Uniformly (w.r.t. $h$ ) Controllable Semidiscrete Schemes}

We adapt [12] to the $2 \mathrm{D}$ case and introduce a family of semidiscrete schemes $\left(\mathcal{W}_{h}^{\alpha, \beta_{1}, \beta_{2}}\right)$ in space, consistent with the homogeneous system in $w$ that we denote by $(\mathcal{W})$. We then determine the possible choices of the parameters $\alpha, \beta_{1}, \beta_{2}$ leading to uniform controllability properties.

\subsection{Semidiscretization of the Wave Equation}

Let us introduce $N \in \mathbb{N}^{*}$ and $h=1 /(N+1)$. We consider the uniform partition of the square $(x, y) \in \Omega$ : $\left(x_{i}, y_{j}\right)=(i h, j h), 0 \leq i, j \leq N+1$ and denote $w_{i j}=w\left(x_{i}, y_{j}\right)$. Let us also consider three parameters $\alpha, \beta_{1}, \beta_{2}$ independent of $h$ such that

$$
\left(\alpha, \beta_{1}, \beta_{2}\right) \in[0,1 / 4] \times \mathbb{R}^{+} \times \mathbb{R}^{+} \text {and } 2 \beta_{1}+\beta_{2} \neq 0
$$

Using the notation $\mathcal{W}_{i j} \in \mathcal{M}_{3 \times 3}(\mathbb{R})$ defined by

$$
\mathcal{W}_{i j}=\left(\begin{array}{ccc}
w_{i-1, j+1} & w_{i, j+1} & w_{i+1, j+1} \\
w_{i-1, j} & w_{i j} & w_{i+1, j} \\
w_{i-1, j-1} & w_{i, j-1} & w_{i+1, j-1}
\end{array}\right), \quad \forall 1 \leq i, j \leq N
$$

we introduce the following finite-dimensional (semidiscrete) approximation of $(\mathcal{W})$ :

$$
\begin{aligned}
\left(\mathcal{W}_{h}^{\alpha, \beta_{1}, \beta_{2}}\right) \quad & \mathcal{M}_{\alpha} \cdot \mathcal{W}_{i j}^{\prime \prime}(t)-\frac{1}{h^{2}} \mathcal{K}_{\beta_{1}, \beta_{2}} \cdot \mathcal{W}_{i j}(t)=0, \quad 1 \leq i, j \leq N, \quad 0 \leq t \leq T, \\
& w_{i 0}(t)=w_{i, N+1}(t)=w_{0 j}(t)=w_{N+1, j}(t)=0, \quad 0 \leq i, j \leq N+1, \quad 0 \leq t \leq T, \\
& \left(w_{i j}(T), w_{i j}^{\prime}(T)\right)=\left(w_{i j}^{0}, w_{i j}^{1}\right), \quad 0 \leq i, j \leq N+1,
\end{aligned}
$$

where

$$
\mathcal{M}_{\alpha}=\left(\begin{array}{ccc}
\alpha^{2} & \alpha(1-2 \alpha) & \alpha^{2} \\
\alpha(1-2 \alpha) & (1-2 \alpha)^{2} & \alpha(1-2 \alpha) \\
\alpha^{2} & \alpha(1-2 \alpha) & \alpha^{2}
\end{array}\right), \quad \mathcal{K}_{\beta_{1}, \beta_{2}}=\frac{1}{2 \beta_{1}+\beta_{2}}\left(\begin{array}{ccc}
\beta_{1} & \beta_{2} & \beta_{1} \\
\beta_{2} & -4\left(\beta_{1}+\beta_{2}\right) & \beta_{2} \\
\beta_{1} & \beta_{2} & \beta_{1}
\end{array}\right) .
$$

The inner product in (3) designates component-by-component multiplication. Straightforward formal Taylor expansions lead to the following proposition.

Proposition 2.1 For all $\left(\alpha, \beta_{1}, \beta_{2}\right) \in[0,1 / 4] \times \mathbb{R}^{+} \times \mathbb{R}^{+}$satisfying $2 \beta_{1}+\beta_{2} \neq 0$, the semidiscrete scheme $\left(\mathcal{W}_{h}^{\alpha, \beta_{1}, \beta_{2}}\right)$ is consistent of order 2 with $(\mathcal{W})$. 
Remark 2.1

- The case $\left(\alpha, \beta_{1}, \beta_{2}\right)=(0,0,1)$ corresponds to the usual (explicit) scheme

$$
\begin{gathered}
\left(\mathcal{W}_{h}^{0,0,1}\right) \quad w_{i j}^{\prime \prime}(t)+\frac{1}{h^{2}}\left(4 w_{i j}(t)-w_{i+1, j}(t)-w_{i-1, j}(t)-w_{i, j+1}(t)-w_{i, j-1}(t)\right)=0 \\
1 \leq i, j \leq N, \quad 0 \leq t \leq T
\end{gathered}
$$

- The implicit semidiscrete scheme $\left(\mathcal{W}_{h}^{\alpha, 0, \beta_{2}}\right)=\left(\mathcal{W}_{h}^{\alpha, 0,1}\right)$ for all $\beta_{2}>0$ corresponds to the usual finitedifference discretization of the equation

$$
\left(I+\alpha h^{2} \partial_{x}^{2}\right)\left(I+\alpha h^{2} \partial_{y}^{2}\right) w^{\prime \prime}-\Delta w=0, \quad \text { in } \quad \Omega \times(0, T) .
$$

- On the other hand, the implicit semidiscrete scheme $\left(\mathcal{W}_{h}^{\alpha, \beta_{1}, 0}\right)=\left(\mathcal{W}_{h}^{\alpha, 1,0}\right)$ for all $\beta_{1}>0$ corresponds to the usual finite-difference discretization of the equation

$$
\left(I+\alpha h^{2} \partial_{x}^{2}\right)\left(I+\alpha h^{2} \partial_{y}^{2}\right) w^{\prime \prime}-\left(I+\alpha h^{2} \partial_{x}^{2}\right) \partial_{y}^{2} w-\left(I+\alpha h^{2} \partial_{y}^{2}\right) \partial_{x}^{2} w=0, \quad \Omega \times(0, T) .
$$

- Reference [9] analyzes the scheme $\left(\mathcal{W}_{h}^{1 / 4,1,1}\right)$ derived from a mixed finite-element approach, that consists of approximating $\left(w, w^{\prime}\right)$ in $\left(\mathbb{Q}_{1}, \mathbb{Q}_{0}\right)$, where $\mathbb{Q}_{k}$ is the space of piecewise polynomials of degree $k$.

Then, introducing the unknown

$$
W_{h}(t)=\left(w_{11}(t), w_{21}(t), \ldots, w_{N 1}(t), \ldots, w_{1 N}(t), w_{2 N}(t), \ldots, w_{N N}(t)\right)^{T} \in \mathbb{R}^{N^{2}}, \forall t \geq 0,
$$

one associates with $\left(\mathcal{W}_{h}^{\alpha, \beta_{1}, \beta_{2}}\right)$ the vectorial form (denoted in the same way)

$$
\begin{array}{rlr}
\left(\mathcal{W}_{h}^{\alpha, \beta_{1}, \beta_{2}}\right) & M^{\alpha} W_{h}^{\prime \prime}(t)+K_{h}^{\beta_{1}, \beta_{2}} W_{h}(t)=0, & 0 \leq t \leq T, \\
& \left(W_{h}(0), W_{h}^{\prime}(0)\right)=\left(W_{h}^{0}, W_{h}^{1}\right),
\end{array}
$$

where

$$
\left(W_{h}^{0}, W_{h}^{1}\right)=\left(w_{i j}^{0}, w_{i j}^{1}\right)_{1 \leq i, j \leq N} \in \mathbb{R}^{2 N^{2}}
$$

are the initial data. The mass and stiffness matrices are denoted by, $M^{\alpha}, K_{h}^{\beta_{1}, \beta_{2}} \in \mathcal{M}_{N^{2} \times N^{2}}(\mathbb{R})$ respectively. These matrices are tridiagonal, block-symmetric and positive definite and take the following forms:

$$
\begin{aligned}
& M^{\alpha}=\left(\begin{array}{cccccc}
A & B & & & & \\
B & A & B & & (0) & \\
& B & \ddots & \ddots & & \\
& & \ddots & \ddots & B & \\
& (0) & & B & A & B \\
& & & & B & A
\end{array}\right)_{N^{2} \times N^{2}} \\
& K_{h}^{\beta_{1}, \beta_{2}}=\frac{1}{h^{2}}\left(\begin{array}{cccccc}
C & D & & & & \\
D & C & D & & (0) & \\
& D & \ddots & \ddots & & \\
& & \ddots & \ddots & D & \\
& (0) & & D & C & D \\
& & & & D & C
\end{array}\right)_{N^{2} \times N^{2}}
\end{aligned}
$$


where $A, B, C, D \in \mathcal{M}_{N \times N}(\mathbb{R})$ are tridiagonal matrices as follows:

$$
\begin{aligned}
& A=\operatorname{tridiag}\left(\alpha(1-2 \alpha),(1-2 \alpha)^{2}, \alpha(1-2 \alpha)\right), \\
& B=\operatorname{tridiag}(\alpha,(1-2 \alpha), \alpha), \\
& C=\left(2 \beta_{1}+\beta_{2}\right)^{-1} \operatorname{tridiag}\left(-\beta_{2}, 4\left(\beta_{1}+\beta_{2}\right),-\beta_{2}\right), \\
& D=\left(2 \beta_{1}+\beta_{2}\right)^{-1} \operatorname{tridiag}\left(-\beta_{1},-\beta_{2},-\beta_{1}\right) .
\end{aligned}
$$

Finally, we associate with (6) the semidiscrete energy

$$
E_{h}^{\alpha, \beta_{1}, \beta_{2}}(t)=\frac{1}{2} h^{2}\left(\left\langle M^{\alpha} W_{h}^{\prime}(t), W_{h}^{\prime}(t)\right\rangle+\left\langle K_{h}^{\beta_{1}, \beta_{2}} W_{h}(t), W_{h}(t)\right\rangle\right)
$$

where $\langle\cdot, \cdot\rangle$ denotes the canonical inner product in $\mathbb{R}^{N^{2}}$.

\subsection{Properties of the Semidiscrete Schemes w.r.t. Controllability}

We take $x_{0}=(0,0)$ so that the support of the control $\Gamma_{0}=\{\vec{x}=(x, y) \in \partial \Omega,(x-1)(y-1)=0\}$. We have the following important result, semidiscrete version of $(2)$.

Proposition 2.2 Uniform Semidiscrete Observability for $\left(\mathcal{W}_{h}^{1 / 4, \beta_{1}, \beta_{2}}\right)$. Let $\left(\alpha, \beta_{1}, \beta_{2}\right) \in\{1 / 4\} \times \mathbb{R}^{+} \times \mathbb{R}^{+}$ satisfy $2 \beta_{1}+\beta_{2} \neq 0$ and let $\left(W_{h}(t)\right)_{h>0}$ be the solution of the system $\left(\mathcal{W}_{h}^{1 / 4, \beta_{1}, \beta_{2}}\right)$ associated with the initial condition $\left(W_{h}^{0}, W_{h}^{1}\right)$. Given T large enough independent of $h$, there exists a constant $C_{T, h} \leq C<\infty$ such that the following inequality holds:

$$
\begin{aligned}
E_{h}^{1 / 4, \beta_{1}, \beta_{2}}(0) \leq C_{T, h} \frac{h}{2}\left\{\int_{0}^{T}\right. & {\left[\left\langle B W_{N, \cdot}^{\prime}, W_{N, \cdot}^{\prime}\right\rangle+\left\langle B W_{\cdot, N}^{\prime}, W_{\cdot, N}^{\prime}\right\rangle\right] d t+} \\
& \left.-\frac{1}{h^{2}} \int_{0}^{T}\left[\left\langle D W_{N ., \cdot}, W_{N, \cdot}\right\rangle+\left\langle D W_{\cdot, N}, W_{\cdot, N}\right\rangle\right] d t\right\}
\end{aligned}
$$

where

$$
W_{N, \cdot}=\left(w_{N, j}\right)_{1 \leq j \leq N} \in \mathbb{R}^{N} \quad \text { and } \quad W_{\cdot, N}=\left(w_{i, N}\right)_{1 \leq i \leq N} \in \mathbb{R}^{N} .
$$

The technical proof of this result, based on semidiscrete multipliers, has been given in detail in [9] for the scheme $\left(\mathcal{W}_{h}^{1 / 4,1,1}\right)$. The proof for the schemes $\left(\mathcal{W}_{h}^{1 / 4, \beta_{1}, \beta_{2}}\right)$, for all $\beta_{1}, \beta_{2} \in \mathbb{R}^{+}$satisfying $2 \beta_{1}+\beta_{2} \neq 0$, is similar. In Appendix A.1, we present a simpler proof in the case $\left(\alpha, \beta_{1}, \beta_{2}\right)=(1 / 4,0,1)$, that is important in the sequel. The uniform semidiscrete observability (7), implies the strong convergence in $L^{2}$ of the discrete sequence of controls $\left\{v_{h}\right\}_{(h>0)}$ associated with $\left(\mathcal{S}_{h}^{1 / 4, \beta_{1}, \beta_{2}}\right)$ towards the HUM control $v$ when $h$ goes to zero (assuming the strong convergence of $\left(Y_{h}^{0}, Y_{h}^{1}\right)$ toward $\left(y^{0}, y^{1}\right)$ in $\left.L^{2}(\Omega) \times H^{-1}(\Omega)\right)$. According to this proposition, the schemes $\left(\mathcal{W}_{h}^{1 / 4,0,1}\right),\left(\mathcal{W}_{h}^{1 / 4,1,0}\right)$ and $\left(\mathcal{W}_{h}^{1 / 4,1,1}\right)$ (highlighted in Remark 2.1) are uniformly controllable. On the other hand, when $\alpha$ independent of $h$ is strictly less than $1 / 4$, the uniform observability of $\left(\mathcal{W}_{h}^{\alpha, \beta_{1}, \beta_{2}}\right)$ does not hold and one may exhibit initial conditions $\left(W_{h}^{0}, W_{h}^{1}\right)$ for which the constant $C_{T, h}$ in $(7)$ blows up when $h$ goes to zero (we refer to [8] for the proof in the case $\left(\mathcal{W}_{h}^{0,0,1}\right)$ ).

Beyond the technical proof of Proposition 2.2 using multipliers, let us explain why the semidiscretization $\left(\mathcal{W}_{h}^{1 / 4, \beta_{1}, \beta_{2}}\right)$ provides a uniform observability property. In order to have the uniform observability property (2), it is necessary to consider $T$ sufficiently large. This is due to the fact that the velocity of plane waves is one and then any perturbation of the initial data will take some time to arrive at the observation zone $\Gamma_{0}$. For semidiscrete schemes, we can define plane waves as solutions of the form

$$
w_{i j}=e^{i\left(\vec{\xi} \cdot\left(x_{i}, x_{j}\right)-\omega_{h} t\right)}, \quad \vec{\xi}=\left(\xi_{1}, \xi_{2}\right),
$$

with $i^{2}=-1$ leading to a relation between the mode $\vec{\xi}$ (or wave number) and the frequency $\omega_{h}$. 
LEMMA 2.1 Let $\left(\alpha, \beta_{1}, \beta_{2}\right) \in[0,1 / 4) \times \mathbb{R}^{+} \times \mathbb{R}^{+}$satisfy $2 \beta_{1}+\beta_{2}>0$. For all $\vec{\xi} \in(-\pi / h, \pi / h)^{2}$, the frequencies for schemes $\left(\mathcal{W}_{h}^{\alpha, \beta_{1}, \beta_{2}}\right)$ are given by

$$
\omega_{h}^{\alpha, \beta_{1}, \beta_{2}}(\vec{\xi})=\frac{2}{h} \sqrt{\frac{2 \beta_{1}\left(s\left(\xi_{1}\right) c\left(\xi_{2}\right)+s\left(\xi_{2}\right) c\left(\xi_{1}\right)\right)+\beta_{2}\left(s\left(\xi_{1}\right)+s\left(\xi_{2}\right)\right)}{\left(2 \beta_{1}+\beta_{2}\right)\left(1-4 \alpha s\left(\xi_{1}\right)\right)\left(1-4 \alpha s\left(\xi_{2}\right)\right)}}
$$

where

$$
s\left(\xi_{i}\right)=\sin ^{2}\left(\xi_{i} h / 2\right), \quad c\left(\xi_{i}\right)=\cos ^{2}\left(\xi_{i} h / 2\right), \quad i=1,2 .
$$

The group velocity associated with a mode $\vec{\xi}$ in the direction $v=\left(v_{1}, v_{2}\right)$ is given by $\nabla_{\xi} \omega_{h} \cdot v$. A necessary condition in order to have a uniform (in $h$ ) observability property in finite time $T>T^{\star}$ is that the group velocity associated with any mode $\vec{\xi}$ is strictly bounded from below by a constant (independent of $\vec{\xi}$ and $h$ ) for at least one direction $v$. Otherwise, some solutions of the semidiscrete system would propagate so slowly in any direction that the observability would require a larger time $T$ as $h \rightarrow 0$. To guarantee that we have a group velocity uniformly bounded from below for at least one direction $v$, it is sufficient to have a uniform bound from below (in $\vec{\xi}$ and $h$ ) for

$$
\left|\nabla_{\xi} \omega_{h}\right|=\sqrt{\left|\partial_{\xi_{1}} \omega_{h}\right|^{2}+\left|\partial_{\xi_{2}} \omega_{h}\right|^{2}}
$$

For the continuous wave equation, $\omega(\vec{\xi})=|\vec{\xi}|$ and therefore $\left|\nabla_{\xi} \omega\right|=1$. For the semidiscrete schemes, computations lead to the following result.

LEMma 2.2 [Bound on the Semidiscrete Group Velocity for $\left.\left(\mathcal{W}_{h}^{\alpha, \beta_{1}, \beta_{2}}\right)\right] \forall \vec{\xi} \in(-\pi / h, \pi / h)^{2}$ ]

- $\forall\left(\alpha, \beta_{1}, \beta_{2}\right) \in[0,1 / 4) \times\left(\mathbb{R}^{+}\right)^{2}, 2 \beta_{1}+\beta_{2}>0, O(h) \leq\left|\nabla_{\xi} \omega_{h}^{\alpha, \beta_{1}, \beta_{2}}(\vec{\xi})\right| \leq 1$;

- $\forall\left(\alpha, \beta_{1}, \beta_{2}\right) \in\{1 / 4\} \times \mathbb{R}^{+} \times \mathbb{R}_{\star}^{+}, 1 \leq\left|\nabla_{\xi} \omega_{h}^{\alpha, \beta_{1}, \beta_{2}}(\vec{\xi})\right| \leq O\left(h^{-3}\right)$,

- $\forall\left(\alpha, \beta_{1}, \beta_{2}\right) \in\{1 / 4\} \times \mathbb{R}_{\star}^{+} \times\{0\}, 1 \leq\left|\nabla_{\xi} \omega_{h}^{\alpha, \beta_{1}, \beta_{2}}(\vec{\xi})\right| \leq O\left(h^{-2}\right)$.

The group velocity of high frequencies associated with $\left(\mathcal{W}_{h}^{\alpha, \beta_{1}, \beta_{2}}\right)$ with $\alpha \in[0,1 / 4)$ is not uniformly bounded from below so that there exist initial conditions for which a uniform observability inequality does not hold. On the contrary, the group velocity for $\alpha=1 / 4$ is bounded from below by 1 , which is the group velocity of the continuous case. This explains why these schemes are likely to provide uniform observability properties. Actually, they do provide the uniform property according to Proposition 2.2, but we do not know if the above spectral condition on $\nabla_{\xi} \omega_{h}$ is sufficient to guarantee a uniform observability inequality. That is why the multiplier technique is used to obtain the result.

Once a uniform controllable semidiscrete scheme is known, the first possibility, in order to obtain an approximation of the control, is to solve exactly in time the finite differential system (6) using a spectral approach (see [13] in a similar context and Appendix A.2). For the square domain where the eigenfunctions and eigenvalues of the matrices $M^{\alpha}$ and $K_{h}^{\beta_{1}, \beta_{2}}$ are explicitly known, the conjugate gradient method is particularly efficient and leads to impressive results. The details are presented in Section 4 and Appendix A.2. The second possibility is to introduce a time approximation that will lead us to the optimal triplet $\left(\alpha, \beta_{1}, \beta_{2}\right)$.

\section{Uniformly Controllable (w.r.t. $h$ and $\Delta t$ ) Fully-Discrete Schemes}

The previous analysis indicates that, with respect to controllability, the relevant choice is $\alpha=1 / 4$. A priori, the additional time approximation does not affect the controllability property. However, the effect on the dispersion property is an important issue in practice; we will now proceed to control it with an appropriate choice of the parameters $\beta_{1}$ and $\beta_{2}$. 


\subsection{Full Discretization of the Wave Equation $(\mathcal{W})$}

A full approximation of $(\mathcal{W})$ is obtained using the leapfrog scheme for the second derivative in time, leading to

$$
\begin{aligned}
& \left(\mathcal{W}_{h, \Delta t}^{\alpha, \beta_{1}, \beta_{2}}\right) \quad M^{\alpha} \frac{W_{h}^{k+1}-2 W_{h}^{k}+W_{h}^{k-1}}{\Delta t^{2}}+K_{h}^{\beta_{1}, \beta_{2}} W_{h}^{k}=0, \quad 0 \leq k \leq K, \\
& W_{h}^{K}=w_{h}^{0}, \quad \frac{W_{h}^{K+1}-W_{h}^{K-1}}{2 \Delta t}=w_{h}^{1},
\end{aligned}
$$

consistent of order 2 in time and space, where $\Delta t$ designates the time 63step and $k$ the time index in $(0, K)$ such that $K \Delta t=T$. The scheme $\left(\mathcal{W}_{h, \Delta t}^{\left.\alpha, \beta_{1}, \beta_{2}\right)}\right.$ is stable under the so-called Courant-Friedrichs-Levy (CFL) condition,

$$
\Delta t^{2} \sup _{\vec{\xi} \in(0, \pi / h)^{2}}\left(\omega_{h}^{\alpha, \beta_{1}, \beta_{2}}(\vec{\xi})\right)^{2} \leq 4 .
$$

Straightforward computations then lead to the following stability conditions and illustrate the balance with the dispersion properties.

LEMMA 3.1 CFL Stability condition for $\left(\mathcal{W}_{h, \Delta t}^{\alpha, \beta_{1}, \beta_{2}}\right)$.

- $\forall\left(\alpha, \beta_{1}, \beta_{2}\right) \in[0,1 / 4) \times\left(\mathbb{R}^{+}\right)^{2}, 2 \beta_{1}+\beta_{2}>0,\left(\mathcal{W}_{h, \Delta t}^{\left.\alpha, \beta_{1}, \beta_{2}\right)}\right.$ is stable under the condition $\Delta t \leq C h$.

- $\forall\left(\alpha, \beta_{1}, \beta_{2}\right) \in\{1 / 4\} \times \mathbb{R}^{+} \times \mathbb{R}_{\star}^{+},\left(\mathcal{W}_{h, \Delta t}^{\left.\alpha, \beta_{1}, \beta_{2}\right)}\right.$ is stable under the condition $\Delta t \leq\left(\pi^{2} / \sqrt{32}\right) h^{3}$.

- $\forall\left(\alpha, \beta_{1}, \beta_{2}\right) \in\{1 / 4\} \times \mathbb{R}_{\star}^{+} \times\{0\},\left(\mathcal{W}_{h, \Delta t}^{\left.\alpha, \beta_{1}, \beta_{2}\right)}\right.$ is stable under the condition $\Delta t \leq(\pi / \sqrt{8}) h^{2}$.

If we exclude the first case for which $\left(\mathcal{W}_{h, \Delta t}^{\alpha, \beta_{1}, \beta_{2}}\right)$ is not uniformly controllable, the other cases lead to restrictive conditions. We remark that the implicit scheme $\left(\mathcal{W}_{h, \Delta t}^{1 / 4,1,0}\right)$ has been studied in [14]. Fortunately, the conditions may be weakened using a Newmark approach, where we replace the term $K_{h}^{\beta_{1}, \beta_{2}} W_{h}^{k}$ in (8) by $1 / 4 K_{h}^{\beta_{1}, \beta_{2}}\left(W_{h}^{k+1}+2 W_{h}^{k}+W_{h}^{k-1}\right)$ in order to obtain the unconditionally stable scheme

$$
\begin{array}{cl}
\left(\mathcal{W}_{\mathcal{N}, h, \Delta t}^{\alpha, \beta_{1}, \beta_{2}}\right) \quad\left(M^{\alpha}+\frac{\Delta t^{2}}{4} K_{h}^{\beta_{1}, \beta_{2}}\right) \frac{W_{h}^{k+1}-2 W_{h}^{k}+W_{h}^{k-1}}{\Delta t^{2}}+K_{h}^{\beta_{1}, \beta_{2}} W_{h}^{k}=0, \quad \forall 0 \leq k \leq K, \\
W_{h}^{K}=w_{h}^{0}, \frac{W_{h}^{K+1}-W_{h}^{K-1}}{2 \Delta t}=w_{h}^{1} .
\end{array}
$$

To conclude, let us now analyze whether this fully-discrete system $\left(\mathcal{W}_{\mathcal{N}, h, \Delta t}^{\left.\alpha, \beta_{1}, \beta_{2}\right)}\right.$ conserves the observability properties of the semidiscrete scheme for some value of the triplet $\left(\alpha, \beta_{1}, \beta_{2}\right)$.

\subsection{Properties of the Fully-Discrete Schemes w.r.t. Controllability}

Following the analysis of Section 2, we study the group velocity of discrete plane waves of the form

$$
w_{i j}^{k}=e^{\vec{i}\left(\vec{\xi} \cdot\left(x_{i}, x_{j}\right)-\omega_{h, \Delta t} k \Delta t\right)} .
$$

For the discrete system (8), the following relation between the mode $\vec{\xi}$ and the frequency $\omega_{h, \Delta t}$ holds:

$$
\omega_{h, \Delta t}^{\alpha, \beta_{1}, \beta_{2}}(\vec{\xi})=\frac{2}{\Delta t} \arcsin \left(\frac{\Delta t}{2} \omega_{h}^{\alpha, \beta_{1}, \beta_{2}}(\vec{\xi})\right)
$$


while for (9), we get

$$
\omega_{\mathcal{N}, h, \Delta t}^{\alpha, \beta_{1}, \beta_{2}}(\vec{\xi})=\frac{2}{\Delta t} \arcsin \left(\frac{\Delta t}{2} \sqrt{\frac{\omega_{h}^{\alpha, \beta_{1}, \beta_{2}}(\vec{\xi})^{2}}{1+\frac{\Delta t^{2}}{4} \omega_{h}^{\alpha, \beta_{1}, \beta_{2}}(\vec{\xi})^{2}}}\right)
$$

The group velocity associated with a mode $\vec{\xi}$ in the direction $v=\left(v_{1}, v_{2}\right)$ is given by $\nabla_{\xi} \omega_{h, \Delta t} \cdot v$ and a necessary condition in order to have a uniform (in $h$ and $\Delta t$ ) observability property in finite time $T>T^{\star}$ is once again to have a uniform bound from below (in $\vec{\xi}, h, \Delta t$ ) for

$$
\left|\nabla_{\xi} \omega_{h, \Delta t}\right|=\sqrt{\left|\partial_{\xi_{1}} \omega_{h, \Delta t}\right|^{2}+\left|\partial_{\xi_{2}} \omega_{h, \Delta t}\right|^{2}}
$$

We obtain the following result.

Lemma 3.2 Bound on the Fully-Discrete Group Velocity

- $\forall\left(\alpha, \beta_{1}, \beta_{2}\right) \in[0,1 / 4) \times \mathbb{R}^{+} \times \mathbb{R}^{+}, 2 \beta_{1}+\beta_{2}>0, \min _{\vec{\xi} \in(-\pi / h, \pi / h)^{2}}\left|\nabla_{\xi} \omega_{h, \Delta t}^{\alpha, \beta_{1}, \beta_{2}}(\vec{\xi})\right|=O(h) ;$

- $\forall\left(\alpha, \beta_{1}, \beta_{2}\right) \in\{1 / 4\} \times \mathbb{R}^{+} \times \mathbb{R}_{\star}^{+}, \min _{\vec{\xi} \in(-\pi / h, \pi / h)^{2}}\left|\nabla_{\xi} \omega_{\mathcal{N}, h, \Delta t}^{\alpha, \beta_{1}, \beta_{2}}(\vec{\xi})\right|=O\left(h^{3 / 2} \Delta t^{-1}\right)$;

- $\forall\left(\alpha, \beta_{1}, \beta_{2}\right) \in\{1 / 4\} \times \mathbb{R}_{\star}^{+} \times\{0\}, \min _{\vec{\xi} \in(-\pi / h, \pi / h)^{2}}\left|\nabla_{\xi} \omega_{\mathcal{N}, h, \Delta t}^{\alpha, \beta_{1}, \beta_{2}}(\vec{\xi})\right|=\min \left(1, \frac{1}{2} h^{2} \Delta t^{-2}\right)+O\left(h^{3} \Delta t^{-2}\right)$.

As expected, the group velocity for $\alpha \in[0,1 / 4)$ vanishes when $h$ and $\Delta t$ go to zero. In particular, the standard five-point scheme $\left(\mathcal{W}_{h, \Delta t}^{0,0,1}\right)$ is not uniformly controllable. On the other hand, for $\alpha=1 / 4$ and $\beta_{2} \neq 0$ (in particular the scheme $\left(\mathcal{W}_{\mathcal{N}, h, \Delta t}^{1 / 4,1,1}\right)$ recently used in [9]), the group velocity is bounded below only if $\Delta t$ is of order of $h^{3 / 2}$. From a practical viewpoint, this condition remains too restrictive. Finally, the group velocity associated with the scheme $\left(\mathcal{W}_{\mathcal{N}, h, \Delta t}^{1 / 4, \beta_{1}, 0}\right)=\left(\mathcal{W}_{\mathcal{N}, h, \Delta t}^{1 / 4,1,0}\right)$ for all $\beta_{1}>0$ is uniformly bounded below if $\Delta t$ and $h$ are of the same order. In particular, when $\Delta t \leq h / \sqrt{2}$, the group velocity is higher for all components than the group velocity associated with the continuous wave equation. The corresponding scheme is then expected to be uniformly controllable with respect to $h$ and $\Delta t$, with $T$ being fixed and large enough.

As a conclusion, the crucial use of the Newmark method leads to the scheme $\left(\mathcal{W}_{\mathcal{N}, h, \Delta t}^{1 / 4,1,0}\right)$, stable under the condition $\Delta t=O(h)$ and expected to be uniformly controllable, that we are looking for. In addition, this scheme is very slightly dispersive with a spectrum $\left\{\omega_{\mathcal{N}, h, \Delta t}^{1 / 4,1,0}(\vec{\xi})\right\}$ very close to the continuous spectrum $\left\{\omega^{2}(\vec{\xi})\right\}_{\xi \in(0, \pi / h)}$ defined by $\omega(\vec{\xi})=|\vec{\xi}|$. For $\Delta t=h / \sqrt{2}$, we have

$$
\omega(\vec{\xi}) \leq \omega_{\mathcal{N}, h, h / \sqrt{2}}^{1 / 4,1,0}(\vec{\xi}) \leq \sqrt{2} \omega(\vec{\xi}), \quad \forall \vec{\xi} \in(-\pi / h, \pi / h)^{2}
$$

and the following remarkable equality for $\vec{\xi}=\left(\xi_{1}, \xi_{1}\right)$ :

$$
\omega\left(\xi_{1}, \xi_{1}\right)=\omega_{\mathcal{N}, h, h / \sqrt{2}}^{1 / 4,1,0}\left(\xi_{1}, \xi_{1}\right)
$$

\section{Numerical Experiments}

We compare now numerically the behavior of the schemes $\left(\mathcal{W}_{h, \Delta t}^{0,0,1}\right),\left(\mathcal{W}_{h}^{1 / 4,0,1}\right),\left(\mathcal{W}_{\mathcal{N}, h, \Delta t}^{1 / 4,0}\right)$. The HUM method reduces the controllability problem to an optimal control one, which consists of solving the linear equation $\Lambda\left(w^{0}, w^{1}\right)=\left(y^{1},-y^{0}\right)$ where $\Lambda$ designates the HUM operator from $H_{0}^{1}(\Omega) \times L^{2}(\Omega)$ into $H^{-1}(\Omega) \times L^{2}(\Omega)$. The HUM control (of minimal $L^{2}$-norm) is given by $v=\nabla w \cdot \vec{\nu}$ (see [1]). The linear equation is solved with a 
conjugate gradient $(\mathrm{CG})$ algorithm (we refer to $[3,10,15]$ ). At each iteration, the state and adjoint systems in $y$ and $w$ are solved using either the semidiscrete scheme $\left(\mathcal{W}_{h}^{1 / 4,0,1}\right)$ or the fully-discrete one $\left(\mathcal{W}_{\mathcal{N}, h, \Delta t}^{1 / 4,1,0}\right)$. The exact resolution in time for $\left(\mathcal{W}_{h}^{1 / 4,0,1}\right)$ is explained in Appendix A.2. A key point of the CG algorithm is the choice of a scalar product on the space $E=H_{0}^{1}(\Omega) \times L^{2}(\Omega)$. At the continuous level, the natural one is

$$
\left\langle\left(f_{1}, f_{2}\right),\left(g_{1}, g_{2}\right)\right\rangle_{E}=\int_{\Omega}\left(\nabla f_{1} \cdot \nabla g_{1}+f_{2} \cdot g_{2}\right) d x, \quad \forall\left(f_{1}, f_{2}\right),\left(g_{1}, g_{2}\right) \in E .
$$

On the discrete space $E_{h}$ of $E$, the scalar product corresponding to $\left(\mathcal{W}_{\mathcal{N}, h, \Delta t}^{\alpha, \beta_{1}, \beta_{2}}\right)$ is

$$
\left\langle\left(f_{1 h}, f_{2 h}\right),\left(g_{1 h}, g_{2 h}\right)\right\rangle_{E_{h}}=h^{2}\left(\left\langle-K_{h}^{\beta_{1}, \beta_{2}} f_{1 h}, g_{1 h}\right\rangle+\left\langle\left(M^{\alpha}+\frac{\Delta t^{2}}{4} K_{h}^{\beta_{1}, \beta_{2}}\right) f_{2 h}, g_{2 h}\right\rangle\right) .
$$

For $\left(\alpha, \beta_{1}, \beta_{2}\right) \in\{1 / 4\} \times \mathbb{R}^{+} \times \mathbb{R}^{+}, 2 \beta_{1}+\beta_{2}>0$, leading to uniform controllable schemes, this scalar product leads to an accurate descent direction and fast convergence. From this point of view, the modification of the usual scheme may be seen as a preconditioning technique for the CG algorithm. In practice, the algorithm is initialized with $\left(w_{h 0}^{0}, w_{h 0}^{1}\right)=(0,0)$ and stopped as soon as the relative residual $r_{k}$ at the $k$ th iteration satisfies $r_{k} \leq \varepsilon r_{0}$, for some $\varepsilon>0$.

\subsection{Discontinuous Initial Condition}

We consider the most singular situation where the initial position $y^{0}$ is discontinuous. Precisely, still on the unit square $(0,1)^{2}$, we define the following functions:

$$
y^{0}(\vec{x})=\left\{\begin{array}{ll}
40, & \vec{x} \in\left(\frac{1}{3}, \frac{2}{3}\right)^{2} \\
0, & \text { elsewhere, }
\end{array} ; \quad y^{1}(\vec{x})=0 .\right.
$$

We assume that the control is active only on two consecutive sides, precisely on $\Gamma_{0}(0,0) \equiv\{\vec{x} \in \partial \Omega,(1-x)(1-$ $y)=0\}$ and take $T=3>2 \sqrt{2}$. With this discontinuous initial position, the usual schemes $\left(\mathcal{W}_{h}^{0,0,1}\right),\left(\mathcal{W}_{h, \Delta t}^{0,0,1}\right)$ completely fail for all $h>0$. On the contrary, the schemes $\left(\mathcal{W}_{h}^{1 / 4,0,1}\right),\left(\mathcal{W}_{\mathcal{N}, h, h \sqrt{2}}^{1 / 4,0,1}\right)$ provide a convergent algorithm after a number of iterations independent of $h$ (see Table 1): the residual varies as $r_{k} / r_{0} \approx O\left(10^{-0.9695 k}\right.$ ) with respect to the iterations. The $L^{2}$-norm of the control converges as $h$ goes to zero (see Table 1 ) and the solution of the semidiscrete wave system is driven to rest at time $T$. These properties are preserved when the time approximation is added (see Table 2). We recall that, for any $v \in H^{-1}(\Omega)$, we have

$$
\|v\|_{H^{-1}(\Omega)}=|u|_{H_{0}^{1}} \equiv\left(\int_{\Omega}|\nabla u|^{2} d x d y\right)^{1 / 2}
$$

where $u \in H_{0}^{1}(\Omega)$ is the solution of the Dirichlet problem: $-\Delta u=v$ in $\Omega, u=0$ on $\partial \Omega$. Figure 1 points out that the initial velocity $w_{h}^{1}$ is discontinuous. The exact controllability of the wave equation associated with (10) is illustrated in Figure 2. Finally, we point out that the Bi-Grid method introduced in [3] fails for the initial condition (10).

\subsection{Behavior of the Schemes $\left(\mathcal{W}_{h}^{1 / 4,0,1}\right)$ and $\left(\mathcal{W}_{\mathcal{N}, h, \Delta t}^{1 / 4,1,0}\right)$ with Respect to $T$}

One may pursue the study of the robustness of the schemes by considering the limit $T \nearrow \infty$. Let us consider the initial condition (10) and assume that $\Gamma_{0}=\partial \Omega$ where $\Omega$ is the unit square domain. Let us denote by $\Lambda_{T}$ the HUM operator $\Lambda$ associated with $T$. It is shown in [16] that

$$
\lim _{T \rightarrow \infty} \frac{\Lambda_{T}}{T}=2\left[\begin{array}{cc}
-\Delta & 0 \\
0 & I
\end{array}\right] .
$$


This result is very useful for validating numerical methods, since it provides the relation

$$
\lim _{T \rightarrow \infty} T\left(w_{T}^{0}, w_{T}^{1}\right)=\left(\chi^{0}, \chi^{1}\right),
$$

where, from $\Lambda_{T}\left(w^{0}, w^{1}\right)=\left(y^{1},-y^{0}\right)$ and (11), $\chi^{0}$ and $\chi^{1}$ are solutions of $\Delta \chi^{0}=y^{1} / 2$ in $\Omega, \chi^{0}=0$ on $\partial \Omega$ and $\chi^{1}=y^{0} / 2$ in $\bar{\Omega}$. The evaluation of $\left(w_{T}^{0}, w_{T}^{1}\right)$ using the scheme $\left(\mathcal{W}_{\mathcal{N}, h, \Delta t}^{1 / 4,1,0}\right)$ may be costly for large values of $T$ if $\Delta t$ is chosen such that $\Delta t \leq h / \sqrt{2}$. Actually, the group velocity of this scheme being bounded from below by $h^{2} \Delta t^{-2} / 2$, all the wave components meet the boundary support $\partial \Omega$ of the control after a time less than or equal to $T$ if

$$
T\left(h^{2} \Delta t^{-2} / 2\right) \geq \operatorname{diam}(\Omega)=\sqrt{2},
$$

leading to the condition $\Delta t \leq h \sqrt{T /(2 \sqrt{2})}$. We refer the reader to [10], where a uniformly controllable scheme is introduced and justified for the 1D wave equation with the condition $\Delta t \leq h \sqrt{T / 2}$. Table 3 gives the difference $T\left(w_{T h}^{0}, w_{T h}^{1}\right)-\left(\chi_{h}^{0}, \chi_{h}^{1}\right)$ obtained with $\left(\mathcal{W}_{\mathcal{N}, h, h / \sqrt{2}}^{1 / 4,1,0}\right)$. The theoretical property (11) is clearly confirmed numerically. We obtain

$$
\left\|T w_{T h}^{0}-\chi_{h}^{0}\right\|_{H_{0}^{1}(\Omega)}=O\left(T^{-1.10}\right) \quad \text { and } \quad\left\|T w_{T h}^{1}-\chi_{h}^{1}\right\|_{L^{2}(\Omega)}=O\left(T^{-1.0087}\right) .
$$

As advocated in [3], these results provide a validation of the numerical methodology introduced here and show that the scheme $\left(\mathcal{W}_{\mathcal{N}, h, \Delta t}^{1 / 4,1,0}\right)$ is particularly robust, accurate, nondissipative and perfectly able to handle very long intervals $[0, T]$. Note that, with the choice $\Delta t=h / \sqrt{2}$, the number of iterations needed to reach convergence decreases with $T$. If

$$
\Delta t \leq h \sqrt{T /(2 \sqrt{2})}
$$

is used, this number is constant and independent of $T$. Moreover, when we use the semidiscrete scheme $\left(\mathcal{W}_{h}^{1 / 4,0,1}\right)$ (see Table 4$)$, we obtain similar results for

$$
\left\|T w_{T h}^{0}-\chi_{h}^{0}\right\|_{H_{0}^{1}(\Omega)}=O\left(T^{-1.14}\right)
$$

and a slightly worse result for

$$
\left\|T w_{T h}^{1}-\chi_{h}^{1}\right\|_{H_{0}^{1}(\Omega)}=O\left(T^{-0.45}\right) .
$$

This provides additional evidence of the robustness of the scheme $\left(\mathcal{W}_{\mathcal{N}, h, \Delta t}^{1 / 4,1}\right)$.

As a conclusion, we point out that the schemes $\left(\mathcal{W}_{h}^{1 / 4,1,0}\right)$ and $\left(\mathcal{W}_{\mathcal{N}, h, \Delta t}^{1 / 4,1,0}\right)$ introduced in this work, uniformly controllable, very slightly dispersive and numerically robust, may be extended to the $3 \mathrm{D}$ case by considering the following modified equation, a consistent approximation of $w^{\prime \prime}-\Delta w=0$,

$$
A_{x}^{h} A_{y}^{h} A_{z}^{h} w^{\prime \prime}-A_{y}^{h} A_{z}^{h} \partial_{x}^{2} w-A_{z}^{h} A_{x}^{h} \partial_{y}^{2} w-A_{x}^{h} A_{y}^{h} \partial_{z}^{2} w=0, \text { in } \Omega \times(0, T),
$$

where

$$
A_{x}^{h}=I+\frac{h^{2}}{4} \partial_{x}^{2}, \quad A_{y}^{h}=I+\frac{h^{2}}{4} \partial_{y}^{2}, \quad A_{z}^{h}=I+\frac{h^{2}}{4} \partial_{z}^{2} .
$$

Following [9], one easily obtains that the associated semidiscrete scheme is uniformly controllable. In addition, the full scheme associated with a Newmark approach is unconditionally stable and has a group velocity uniformly bounded by below if $\Delta t=O(h)$. We refer the reader to [17] for a detailed version of this work including several numerical experiments. 


\section{A Appendix}

\section{A.1 Uniform Observability of the Scheme $\left(\mathcal{W}_{h}^{1 / 4,0,1}\right)$}

We prove Proposition 2.2 in the case $\left(\alpha, \beta_{1}, \beta_{2}\right)=(1 / 4,0,1)$ for which the inequality $(7)$ becomes

$$
\begin{aligned}
E_{h}^{1 / 4,0,1}(0) \leq & C(T)\left\{\frac{h^{3}}{2} \int_{0}^{T}\left[\sum_{i=0}^{N}\left(\frac{w_{i, N}^{\prime}+w_{i+1, N}^{\prime}}{2 h}\right)^{2}+\sum_{j=0}^{N}\left(\frac{w_{N, j}^{\prime}+w_{N, j+1}^{\prime}}{2 h}\right)^{2}\right] d t+\right. \\
& \left.+\frac{h}{2} \int_{0}^{T}\left[\sum_{j=1}^{N} \frac{w_{N, j}}{h} \frac{w_{N, j}}{h}+\sum_{i=1}^{N} \frac{w_{i, N}}{h} \frac{w_{i, N}}{h}\right] d t\right\} .
\end{aligned}
$$

To simplify the notation we write

$$
\begin{gathered}
a_{i j}^{k l}=w_{i k}+w_{i l}+w_{j k}+w_{j l}, \\
b_{i j}^{k l}=w_{i k}^{\prime}+w_{i l}^{\prime}+w_{j k}^{\prime}+w_{j l}^{\prime}, \\
c_{i j}^{k l}=w_{i k}^{\prime \prime}+w_{i l}^{\prime \prime}+w_{j k}^{\prime \prime}+w_{j l}^{\prime \prime}, \\
\Delta_{(1,0)} w_{i j}=2 w_{i j}-w_{i+1, j}-w_{i-1, j}, \\
\Delta_{(0,1)} w_{i j}=2 w_{i j}-w_{i, j+1}-w_{i, j-1} .
\end{gathered}
$$

When multiplying the discrete system by the discrete version of the usual continuous multiplier $(x, y) \cdot \nabla u$, i.e.

$$
(i h, j h) \cdot\left(\frac{w_{i+1, j}-w_{i-1, j}}{2 h}, \frac{w_{i, j+1}-w_{i-1, j}}{2 h}\right)=i \frac{w_{i+1, j}-w_{i-1, j}}{2}+j \frac{w_{i, j+1}-w_{i, j-1}}{2} \equiv \frac{m_{i j}}{2},
$$

and summing over $i$ and $j$, we obtain

$$
0=\frac{h^{2}}{4} \underbrace{\int_{0}^{T} \sum_{i, j=1}^{N}\left(c_{i, i+1}^{j, j+1}+c_{i, i+1}^{j-1, j}+c_{i-1, i}^{j, j+1}+c_{i-1, i}^{j-1, j}\right) m_{i j} d t}_{\equiv C}+\int_{0}^{T} \underbrace{\sum_{i, j=1}^{N}\left(\Delta_{(1,0)} w_{i j}+\Delta_{(0,1)} w_{i j}\right) m_{i j}}_{\equiv D} d t
$$

We study separately $C$ and $D$. Integration by parts in $C$ allows us to obtain

$$
C=\int_{0}^{T} C_{1} d t+\left[C_{2}\right]_{0}^{T}
$$

where

$$
\left(C_{1}, C_{2}\right)=\sum_{i, j=1}^{N}\left(b_{i, i+1}^{j, j+1}+b_{i, i+1}^{j-1, j}+b_{i-1, i}^{j, j+1}+b_{i-1, i}^{j-1, j}\right)\left(-m_{i j}^{\prime}, m_{i j}\right) .
$$

We first consider the term $C_{1}$ above. In order to have the common factor $b_{i, i+1}^{j, j+1}$, we change the indices in the last three terms of $C_{1}$. Then, taking into account that

$$
w_{i, 0}=w_{i, N+1}=w_{0, j}=w_{j, N+1}=0
$$

and after simplification, we obtain

$$
C_{1}=2 \sum_{i, j=0}^{N}\left(b_{i, i+1}^{j, j+1}\right)^{2}-(N+1)\left[\sum_{i=1}^{N}\left(w_{i, N}^{\prime}+w_{i+1, N}^{\prime}\right)^{2}+\sum_{j=1}^{N}\left(w_{N, j}^{\prime}+w_{N, j+1}^{\prime}\right)^{2}\right] .
$$


We now analyze the term $D$. We provide only details for the first term in $D$, which becomes

$$
\sum_{i, j=1}^{N} \Delta_{(1,0)} w_{i j} m_{i j}=\sum_{i, j=1}^{N} \Delta_{(1,0)} w_{i j}\left[i\left(w_{i+1, j}-w_{i-1, j}\right)+j\left(w_{i, j+1}-w_{i, j-1}\right)\right] .
$$

We consider separately these two terms. For the second one, we have

$$
\begin{gathered}
\Delta_{(1,0)} w_{i j} j\left(w_{i, j+1}-w_{i, j-1}\right)=\sum_{i, j=1}^{N} j\left(w_{i j}-w_{i-1, j}\right) w_{i, j+1}-\sum_{i, j=1}^{N} j\left(w_{i+1, j}-w_{i j}\right) w_{i, j+1} \\
-\left[\sum_{i, j=1}^{N} j\left(w_{i j}-w_{i-1, j}\right) w_{i, j-1}-\sum_{i, j=1}^{N} j\left(w_{i+1, j}-w_{i j}\right) w_{i, j-1}\right] .
\end{gathered}
$$

Changing the indices to obtain the common factor $w_{i+1, j}-w_{i j}$ in all the terms and taking into account that

$$
w_{i, 0}=w_{i, N+1}=w_{0, j}=w_{j, N+1}=0,
$$

we obtain

$$
\begin{aligned}
& \sum_{i, j=0}^{N}\left[j\left(w_{i+1, j}-w_{i j}\right)\left(w_{i+1, j+1}-w_{i, j+1}\right)-j\left(w_{i+1, j}-w_{i j}\right)\left(w_{i+1, j-1}-w_{i, j-1}\right)\right] \\
& \quad=-\sum_{i, j=0}^{N}\left(w_{i+1, j+1}-w_{i, j+1}\right)\left(w_{i+1, j}-w_{i j}\right) .
\end{aligned}
$$

An analogous argument allows us to simplify the first term in (16). We finally have

$$
\begin{aligned}
D= & -\sum_{i, j=0}^{N}\left[\left(w_{i+1, j+1}-w_{i+1, j}\right)\left(w_{i, j+1}-w_{i j}\right)+\left(w_{i+1, j+1}-w_{i, j+1}\right)\left(w_{i+1, j}-w_{i j}\right)\right] \\
& +\sum_{i, j=0}^{N}\left[\left(w_{i+1, j}-w_{i j}\right)^{2}+\left(w_{i, j+1}-w_{i j}\right)^{2}\right]-(N+1) \sum_{j=0}^{N}\left(w_{N, j}\right)^{2}-(N+1) \sum_{i=0}^{N}\left(w_{i, N}\right)^{2} .
\end{aligned}
$$

By the Young inequality we can estimate the first term in this formula,

$$
\begin{aligned}
& \sum_{i, j=0}^{N}\left[\left(w_{i+1, j+1}-w_{i+1, j}\right)\left(w_{i, j+1}-w_{i j}\right)+\left(w_{i+1, j+1}-w_{i, j+1}\right)\left(w_{i+1, j}-w_{i j}\right)\right] \\
& \quad \leq \sum_{i, j=0}^{N}\left[\left(w_{i, j+1}-w_{i j}\right)^{2}+\left(w_{i+1, j}-w_{i j}\right)^{2}\right]
\end{aligned}
$$

and then conclude that

$$
D \geq-(N+1)\left(\sum_{j=0}^{N} w_{N, j}^{2}+\sum_{i=0}^{N} w_{i, N}^{2}\right)
$$

Substituting (15) and (18) into (13), we obtain

$$
\begin{aligned}
h^{2} \int_{0}^{T} \sum_{i, j=0}^{N}\left(\frac{b_{i, i+1}^{j, j+1}}{4}\right)^{2} d t \leq & \frac{h}{8} \int_{0}^{T}\left[\sum_{i=1}^{N}\left(\frac{w_{i, N}^{\prime}+w_{i+1, N}^{\prime}}{2}\right)^{2}+\sum_{j=1}^{N}\left(\frac{w_{N, j}^{\prime}+w_{N, j+1}^{\prime}}{2}\right)^{2}\right] d t \\
& +\frac{1}{2} \int_{0}^{T}\left[\sum_{j=1}^{N} w_{N, j} \frac{w_{N, j}}{h}+\sum_{i=1}^{N} w_{i, N} \frac{w_{i, N}}{h}\right] d t-\frac{h^{2}}{4}\left[C_{2}\right]_{0}^{T} .
\end{aligned}
$$


We observe that the term in the left hand side contains only one part of the energy. In order to obtain the full energy, we make an equipartition of the energy. The following lemma is a discrete version of the well-known equipartition of energy for the continuous wave equation, which reads

$$
\left.0=-\int_{0}^{T} \int_{\Omega}\left(\left|w_{t}\right|^{2}+|\nabla u|^{2}\right) d x d t+\int_{\Omega}\left|w_{t} u\right|^{2} d x\right]_{0}^{T}
$$

LEMMA A.1 The following holds:

$$
\begin{aligned}
0= & -h^{2} \int_{0}^{T}\left[\sum_{i, j=0}^{N}\left(\frac{b_{i, i+1}^{j, j+1}}{4}\right)^{2}\right] d t+h^{2}\left[\sum_{i, j=0}^{N}\left(\frac{a_{i, i+1}^{j, j+1}}{4}\right)\left(\frac{b_{i, i+1}^{j, j+1}}{4}\right)\right]_{0}^{T} \\
& +h^{2} \sum_{i, j=0}^{N} \int_{0}^{T}\left[\left(\frac{w_{i+1, j}-w_{i j}}{h}\right)^{2}+\left(\frac{w_{i, j+1}-w_{i j}}{h}\right)^{2}\right] d t
\end{aligned}
$$

The proof of this lemma is straightforward following the idea of the continuous system. When applying Lemma A.1 to the identity (19), we obtain

$$
\begin{aligned}
\int_{0}^{T} E_{h}^{1 / 4,0,1}(t) d t & +\frac{h^{2}}{4}\left[\sum_{i, j=0}^{N} a_{i, i+1}^{j, j+1} b_{i, i+1}^{j, j+1}+C_{2}\right]_{0}^{T} \\
& \leq \frac{h}{8} \int_{0}^{T}\left[\sum_{i=1}^{N}\left(\frac{w_{i, N}^{\prime}+w_{i+1, N}^{\prime}}{2}\right)^{2}+\sum_{j=1}^{N}\left(\frac{w_{N, j}^{\prime}+w_{N, j+1}^{\prime}}{2}\right)^{2}\right] d t \\
& +\frac{1}{2} \int_{0}^{T}\left[\sum_{j=1}^{N} w_{N, j} \frac{w_{N, j}}{h}+\sum_{i=1}^{N} w_{i, N} \frac{w_{i, N}}{h}\right] d t
\end{aligned}
$$

The following lemma allows us to estimate the second term in the left-hand side of this formula.

LEMMA A.2 [see [9]].The following result holds:

$$
h^{2}\left[\sum_{i, j=0}^{N} a_{i, i+1}^{j, j+1} b_{i, i+1}^{j, j+1}+C_{2}\right]_{0}^{T} \leq 8 \sqrt{3} E_{h}^{1 / 4,0,1}(0) .
$$

Then, Lemma A.2 and the conservation of the discrete energy combined with (21) provides

$$
\begin{aligned}
(T-2 \sqrt{3}) E_{h}^{1 / 4,0,1}(0) \leq & \frac{h}{8} \int_{0}^{T}\left[\sum_{i=1}^{N}\left(\frac{w_{i, N}^{\prime}+w_{i+1, N}^{\prime}}{2}\right)^{2}+\sum_{j=1}^{N}\left(\frac{w_{N, j}^{\prime}+w_{N, j+1}^{\prime}}{2}\right)^{2}\right] d t \\
& +\frac{1}{2} \int_{0}^{T}\left[\sum_{j=1}^{N} w_{N, j} \frac{w_{N, j}}{h}+\sum_{i=1}^{N} w_{i, N} \frac{w_{i, N}}{h}\right] d t
\end{aligned}
$$

\section{A.2 Exact Resolution in Time of $\left(\mathcal{W}_{h}^{1 / 4,0,1}\right)$}

We detail the exact resolution of the two semidiscrete wave systems which appear at each iteration of the conjugate gradient algorithm. The HUM method reduces the exact controllability problem to the determination of the initial conditions $\left(w^{0}, w^{1}\right)$ of $(\mathcal{W})$ such that the solution $\psi$ of the equation $(\mathcal{S})$ satisfies 
$\left(\psi(0), \psi^{\prime}(0)\right)=\left(y^{0}, y^{1}\right)$. The control is then $v=\partial \phi / \partial \nu$ on $\Gamma_{0}$. We consider the semidiscrete scheme $\left(\mathcal{W}_{h}^{1 / 4,0,1}\right)$ and first present the resolution of the semidiscrete system

$$
\begin{aligned}
& M^{1 / 4} \varphi_{h}^{\prime \prime}(t)+K_{h}^{0,1} \varphi_{h}(t)=0, \quad t \in(0, T), \\
& \left(\varphi_{h}(0), \varphi_{h}^{\prime}(0)\right)=\left(w_{h}^{0}, w_{h}^{1}\right)
\end{aligned}
$$

Let us designate by $V_{h}, D_{h} \in \mathcal{M}_{N^{2} \times N^{2}}(\mathbb{R})$ the eigenvector matrix and eigenvalue matrix respectively of $\left(M^{1 / 4}\right)^{-1} K_{h}^{0,1}$ such that $K_{h}^{0,1} V_{h}=M^{1 / 4} V_{h} D_{h}$. The matrix $D_{h}=\left(\lambda_{k}\right)_{1 \leq k \leq N^{2}}$ is diagonal whereas the matrix $V_{h}$ is symmetric and orthonormal such that $V_{h}^{2}=I_{N^{2} \times N^{2}}$. Therefore, the solution $\varphi_{h}(t)=\left(\varphi_{i}(t)\right)_{\left(1 \leq i \leq N^{2}\right)}$ is

$$
\varphi_{i}(t)=\sum_{k=1}^{N^{2}} V_{i k}\left(a_{k} \cos \left(\sqrt{\lambda_{k}} t\right)+\frac{b_{k}}{\sqrt{\lambda_{k}}} \sin \left(\sqrt{\lambda_{k}} t\right)\right), \quad 1 \leq i \leq N^{2}, t \geq 0
$$

where

$$
\left(a_{h}, b_{h}\right)=\left(V_{h}^{-1} w_{h}^{0}, V_{h}^{-1} w_{h}^{1}\right) \quad \text { and } \quad a_{h}=\left(a_{k}\right)_{\left(1 \leq k \leq N^{2}\right)}, b_{h}=\left(b_{k}\right)_{\left(1 \leq k \leq N^{2}\right)} .
$$

On the other hand, the semidiscretization of $(\mathcal{S})$ takes the following form

$$
\begin{aligned}
& M^{1 / 4} \psi_{h}^{\prime \prime}(t)+K_{h}^{0,1}, \psi_{h}(t)=F_{h}\left(\varphi_{h}(t)\right), \quad t \in(0, T), \\
& \left(\psi_{h}(T), \psi_{h}^{\prime}(T)\right)=(0,0),
\end{aligned}
$$

where the vector $F_{h} \in \mathbb{R}^{N^{2}}$ may be easily deduced of the form

$$
F_{h}\left(\varphi_{h}(t)\right)=A^{1} \varphi_{h}(t)+B^{1} \varphi_{h}^{\prime \prime}(t)
$$

with two matrices $A^{1}$ and $B^{1} \in \mathcal{M}_{N^{2} \times N^{2}}$. From (22), the variable $z_{h}(t)=V_{h}^{-1} \psi_{h}(t)$ is the solution of

$$
\begin{aligned}
& z_{h}^{\prime \prime}(t)+D_{h} z_{h}(t)=\left(M^{1 / 4} V_{h}\right)^{-1}\left[A^{1} \varphi_{h}(t)+B^{1} \varphi_{h}^{\prime \prime}(t)\right], \quad t \in(0, T), \\
& z_{h}(T)=0, z_{h}^{\prime}(T)=0 .
\end{aligned}
$$

Let us introduce $P=\left(M^{1 / 4} V_{h}\right)^{-1}$ such that the $j$ th component of the vector $z_{h}$ is the solution of

$$
z_{j}^{\prime \prime}(t)+\lambda_{j} z_{j}(t)=\left(P\left[A^{1} \varphi_{h}(t)+B^{1} \varphi_{h}^{\prime \prime}(t)\right]\right)_{j}, \quad t \geq 0, \quad 1 \leq j \leq N^{2} .
$$

Using (23), the right-hand term is

$$
\left(P\left[A^{1} \varphi_{h}(t)+B^{1} \varphi_{h}^{\prime \prime}(t)\right]\right)_{j}=\sum_{1 \leq k, l, p \leq N^{2}} P_{j k}\left(A_{k l}^{1} V_{l p}-B_{k l}^{1} V_{l p} \lambda_{p}\right)\left(a_{p} \cos \left(\sqrt{\lambda_{p}} t\right)+\frac{b_{p}}{\sqrt{\lambda_{p}}} \sin \left(\sqrt{\lambda_{p}} t\right)\right) .
$$

Consequently, the component $z_{j}$ is the solution of the following system:

$$
z_{j}^{\prime \prime}(t)+\lambda_{j} z_{j}(t)=\sum_{1 \leq p \leq N^{2}} \mathcal{B}_{j p}\left(a_{p} \cos \left(\sqrt{\lambda_{p}} t\right)+\frac{b_{p}}{\sqrt{\lambda_{p}}} \sin \left(\sqrt{\lambda_{p}} t\right)\right), \quad 1 \leq j \leq N^{2}
$$

where

$$
\mathcal{B}=P\left(A^{1} V_{h}-B^{1} V_{h} D_{h}\right)
$$


Some computations lead to

$$
\begin{aligned}
z_{j}(t) & =C_{1}^{j} \cos \left(\sqrt{\lambda_{j}} t\right)+C_{2}^{j} \sin \left(\sqrt{\lambda_{j}} t\right)+\sum_{1 \leq p \leq N^{2}, \lambda_{p} \neq \lambda_{j}} \frac{\mathcal{B}_{j p}}{\lambda_{j}-\lambda_{p}}\left(a_{p} \cos \left(\sqrt{\lambda_{p}} t\right)+\frac{b_{p}}{\sqrt{\lambda_{p}}} \sin \left(\sqrt{\lambda_{p}} t\right)\right) \\
& +\frac{1}{2} \sum_{1 \leq p \leq N^{2}, \lambda_{p}=\lambda_{j}} \frac{\mathcal{B}_{j p}}{\lambda_{p}}\left(\left(a_{p}-b_{p} t\right) \cos \left(\sqrt{\lambda_{p}} t\right)+a_{p} \sqrt{\lambda_{p}} t \sin \left(\sqrt{\lambda_{p}} t\right)\right) \\
& \equiv C_{1}^{j} \cos \left(\sqrt{\lambda_{j}} t\right)+C_{2}^{j} \sin \left(\sqrt{\lambda_{j}} t\right)+F_{j}(t) .
\end{aligned}
$$

We then use the condition at time $T$ in (25) to fix the constants $C_{1}^{j}$ and $C_{2}^{j}$, for $1 \leq j \leq N^{2}$;

$$
\begin{aligned}
& C_{1}^{j}=-\cos \left(\sqrt{\lambda_{j}} T\right) F_{j}(T)+\frac{\sin \left(\sqrt{\lambda_{j}} T\right)}{\sqrt{\lambda_{j}}} F_{j}^{\prime}(T), \\
& C_{2}^{j}=-\sin \left(\sqrt{\lambda_{j}} T\right) F_{j}(T)-\frac{\cos \left(\sqrt{\lambda_{j}} T\right)}{\sqrt{\lambda_{j}}} F_{j}^{\prime}(T) .
\end{aligned}
$$

Finally, we obtain the vector $\psi_{h}(t)=V_{h} z_{h}(t)$ for all $t \in(0, T)$. Then, putting $t=0$ in (26), we obtain

$$
z_{j}(0)=C_{1}^{j}+\sum_{1 \leq p \leq N^{2}, \lambda_{p} \neq \lambda_{j}} \frac{\mathcal{B}_{j p}}{\lambda_{j}-\lambda_{p}} a_{p}+\frac{1}{2} \sum_{1 \leq p \leq N^{2}, \lambda_{p}=\lambda_{j}} \frac{\mathcal{B}_{j p}}{\lambda_{p}} a_{p}
$$

and a similar relation for $z_{j}^{\prime}(0)$. This provides the explicit linear relation between the initial condition $\left(w_{h}^{0}, w_{h}^{1}\right)=\left(V_{h} a_{h}, V_{h} b_{h}\right)$ and $\left(\psi_{h}(0), \psi_{h}^{\prime}(0)\right)=\left(V_{h} z_{h}(0), V_{h} z_{h}^{\prime}(0)\right)=\left(y_{h}^{0}, y_{h}^{1}\right)$. The resulting linear system $\Lambda_{h}\left(w_{h}^{0}, w_{h}^{1}\right):=\left(y_{h}^{0}, y_{h}^{1}\right)$ is efficiently solved using a conjugate gradient algorithm initialized by $\left(w_{h}^{0}, w_{h}^{1}\right)=(0,0)$.

\section{References}

[1] Lions J.-L., Contrôlabilité exacte, perturbations et stabilisation de systèmes distribués, Tome 1, Masson, Paris, 1988.

[2] Russell D. L., Controllability and stabilization theory for linear partial differential equations: recent progress and open questions, SIAM Review, 20(4) (1978), 639-737.

[3] Glowinski R., Li C. H. and Lions J.-L., A numerical approach to the exact boundary controllability of the wave equation (I). Dirichlet controls: Description of the numerical methods, Jap. J. Appl. Math. 7 (1990), 1-76.

[4] Zuazua E., Propagation, Observation, Control and Numerical Approximation of Waves, SIAM Review, 47(2), (2005), 197-243.

[5] Glowinski R., Ensuring well-posedness by analogy; Stokes problem and boundary control for the wave equation, J. Comput. Phys., 103 (1991), 189-221.

[6] Loreti P. and Mehrenberger M., An Ingham type proof for a two-grid observability theorem, ESAIM: COCV, 3 (2008), 604-631.

[7] Glowinski R., Kinton W. and Wheeler M. F., A mixed finite element formulation for the boundary controllability of the wave equation, Int. J. Numer. Methods Eng. 27(3) (1989) , 623-636. 
[8] Zuazua E., Boundary observability for the finite difference space semi-discretizations of the 2-D wave equation in the square, J. Math. Pures Appl., 78, (1999), 523-563.

[9] Castro C., Micu S. and Münch A., Numerical approximation of the boundary control of the 2-D wave equation with mixed finite elements, IMA J. Numerical Analysis. 28(1) (2008), 186-214.

[10] Münch A., A uniformly controllable and implicit scheme for the 1-D wave equation, Mathematical Modelling and Numerical Analysis, 39(2) (2005), 377-418.

[11] Krenk S., Dispersion-corrected explicit integration of the wave equation, Comput. Methods. Appl. Mech. Engrg., 191 (2001), 975-987.

[12] Münch A., Family of implicit schemes uniformly controllable for the 1-D wave equation, C.R.Acad. Sci. Paris, 339 (2004), 733-738.

[13] Bourquin F., Numerical methods for the control of flexible structures, J. Struct. Control, 8(2001).

[14] Rasmussen J. M., Boundary control of linear evolution PDEs : Continuous and Discrete, PhD Thesis, Kongens Lyngby, Denmark, (2004).

[15] Asch M. and Lebeau G., Geometrical aspects of exact boundary controllability for the wave equation - A numerical study, ESAIM: COCV, 3 (1998), 163-212.

[16] Bensoussan A., On the general theory of exact controllability for skew symmetric operators, Acta Appl. Math., 20(3) (1990),197-229.

[17] Asch M. and Münch A., Uniform controllable schemes for the 2-D wave equation, Internal report 4/08, Université de Franche-Comté, 2008. 


\section{List of Tables}

1 Results obtained with $\left(\mathcal{W}_{h}^{1 / 4,0,1}\right)$ where $\Gamma_{0}(0,0) \subset \partial \Omega, T=3, \varepsilon=10^{-7} \ldots \ldots \ldots \ldots$

2 Results obtained with $\left(\mathcal{W}_{\mathcal{N}, h, \Delta t}^{1 / 4,1,0}\right)$ with $\Delta t=h / \sqrt{2}, \Gamma_{0}=\Gamma_{0}(0,0) \subset \partial \Omega, T=3, \varepsilon=10^{-8}$, $\left\|\psi_{h}^{\prime}(0)-y^{1}\right\|_{H^{-1}(\Omega)}=O\left(h^{0.394}\right), \frac{\left\|\psi_{h}(0)-y^{0}\right\|_{L^{2}(\Omega)}}{\left\|y^{0}\right\|_{L^{2}(\Omega)}}=O\left(h^{0.167}\right) \ldots \ldots \ldots \ldots \ldots \ldots$

$3 \quad\left(\mathcal{W}_{\mathcal{N}, h, h / \sqrt{2}}^{1 / 4,1}\right)-\left\|T w_{T h}^{0}-\chi_{h}^{0}\right\|_{L^{2}(\Omega)}$ and $\left\|T w_{T h}^{1}-\chi_{h}^{1}\right\|_{L^{2}(\Omega)}$ vs. $T$ with $\varepsilon=10^{-8}, h=1 / 64 . \ldots 21$ $4 \quad\left(\mathcal{W}_{h}^{1 / 4,1,0}\right)-\left\|T w_{T h}^{0}-\chi_{h}^{0}\right\|_{L^{2}(\Omega)}$ and $\left\|T w_{T h}^{1}-\chi_{h}^{1}\right\|_{L^{2}(\Omega)}$ vs. $T$ with $\varepsilon=10^{-8}, h=1 / 64 \ldots . .22$ 


\begin{tabular}{c|cccc}
\hline$\left(\mathcal{W}_{h}^{1 / 4,0,1}\right)$ & $h=1 / 8$ & $h=1 / 16$ & $h=1 / 32$ & $h=1 / 64$ \\
\hline CG iterations & 8 & 8 & 8 & 8 \\
$\left\|w_{h}^{0}\right\|_{H_{0}^{1}(\Omega)}$ & $9.99 \times 10^{-1}$ & 1.036 & 1.185 & 1.177 \\
$\left\|w_{h}^{1}\right\|_{L^{2}(\Omega)}$ & 5.563 & 5.507 & 5.019 & 4.914 \\
$\left\|v_{h}\right\|_{L^{2}\left(\Gamma_{0} \times(0, T)\right)}$ & $1.407 \times 10^{1}$ & $1.396 \times 10^{1}$ & $1.271 \times 10^{1}$ & $1.246 \times 10^{1}$ \\
$E_{h}^{1 / 4,0,1}(T) / E_{h}^{1 / 4,0,1}(0)$ & $2.25 \times 10^{-7}$ & $6.89 \times 10^{-7}$ & $5.94 \times 10^{-7}$ & $5.60 \times 10^{-7}$ \\
\hline
\end{tabular}

Table 1: Results obtained with $\left(\mathcal{W}_{h}^{1 / 4,0,1}\right)$ where $\Gamma_{0}(0,0) \subset \partial \Omega, T=3, \varepsilon=10^{-7}$. 


\begin{tabular}{c|ccccc}
\hline$\left(\mathcal{W}_{\mathcal{N}, h, \Delta t}^{1 / 4,1,0}\right)$ & $h=1 / 8$ & $h=1 / 16$ & $h=1 / 32$ & $h=1 / 64$ & $h=1 / 128$ \\
\hline CG iterations & 35 & 20 & 18 & 17 & 16 \\
$\left\|\psi_{h}(0)-y^{0}\right\|_{L^{2}(\Omega)}$ & $1.30 \times 10^{-2}$ & $1.14 \times 10^{-2}$ & $1.14 \times 10^{-2}$ & $1.09 \times 10^{-2}$ & $9.76 \times 10^{-3}$ \\
$\left\|y^{0}\right\|_{L^{2}(\Omega)}$ & $1.04 \times 10^{-2}$ & $1.51 \times 10^{-2}$ & $8.55 \times 10^{-3}$ & $6.24 \times 10^{-3}$ & $5.56 \times 10^{-3}$ \\
$\left\|\psi_{h}^{\prime}(0)-y^{1}\right\|_{H^{-1}(\Omega)}$ & 1.242 & 1.259 & 1.233 & 1.234 & 1.232 \\
$\left\|w_{h}^{0}\right\|_{H_{0}^{1}(\Omega)}$ & 5.526 & 5.199 & 4.919 & 4.482 & 4.847 \\
$\left\|w_{h}^{1}\right\|_{L^{2}(\Omega)}$ & $1.338 \times 10^{1}$ & $1.284 \times 10^{1}$ & $1.224 \times 10^{1}$ & $1.238 \times 10^{1}$ & $1.213 \times 10^{1}$ \\
$\left\|v_{h}\right\| L_{L^{2}\left(\Gamma_{0} \times(0, T)\right)}$ & 1.238
\end{tabular}

Table 2: Results obtained with $\left(\mathcal{W}_{\mathcal{N}, h, \Delta t}^{1 / 4,1,0}\right)$ with $\Delta t=h / \sqrt{2}, \Gamma_{0}=\Gamma_{0}(0,0) \subset \partial \Omega, T=3, \varepsilon=10^{-8}, \| \psi_{h}^{\prime}(0)-$ $y^{1} \|_{H^{-1}(\Omega)}=O\left(h^{0.394}\right), \frac{\left\|\psi_{h}(0)-y^{0}\right\|_{L^{2}(\Omega)}}{\left\|y^{0}\right\|_{L^{2}(\Omega)}}=O\left(h^{0.167}\right)$. 


\begin{tabular}{|c|c|c|c|c|c|}
\hline Time & $T=3$ & $T=5$ & $T=10$ & $T=20$ & $T=40$ \\
\hline \hline \# of CG iterations & 13 & 9 & 8 & 8 & 5 \\
\hline$\left\|T w_{T h}^{0}-\chi_{h}^{0}\right\|_{H_{0}^{1}(\Omega)}$ & $7.15 \times 10^{-1}$ & $3.4 \times 10^{-1}$ & $1.40 \times 10^{-1}$ & $1.11 \times 10^{-1}$ & $3.3 \times 10^{-2}$ \\
\hline$\left\|T w_{T h}^{1}-\chi_{h}^{1}\right\|_{L^{2}(\Omega)}$ & $4.12 \times 10^{-1}$ & $2.21 \times 10^{-1}$ & $1.55 \times 10^{-1}$ & $8.46 \times 10^{-2}$ & $2.47 \times 10^{-2}$ \\
\hline
\end{tabular}

Table 3: $\left(\mathcal{W}_{\mathcal{N}, h, h / \sqrt{2}}^{1 / 4,1,0}\right)-\left\|T w_{T h}^{0}-\chi_{h}^{0}\right\|_{L^{2}(\Omega)}$ and $\left\|T w_{T h}^{1}-\chi_{h}^{1}\right\|_{L^{2}(\Omega)}$ vs. $T$ with $\varepsilon=10^{-8}, h=1 / 64$. 


\begin{tabular}{|c|c|c|c|c|c|}
\hline \multicolumn{1}{|c|}{ Time } & $T=3$ & $T=5$ & $T=10$ & $T=20$ & $T=40$ \\
\hline \hline \# of CG iterations & 6 & 6 & 5 & 5 & 4 \\
\hline$\left\|T w_{T h}^{0}-\chi_{h}^{0}\right\|_{H_{0}^{1}(\Omega)}$ & $6.63 \times 10^{-1}$ & $3.48 \times 10^{-1}$ & $1.23 \times 10^{-1}$ & $1.21 \times 10^{-1}$ & $1.97 \times 10^{-2}$ \\
\hline$\left\|T w_{T h}^{1}-\chi_{h}^{1}\right\|_{L^{2}(\Omega)}$ & $3.70 \times 10^{-1}$ & $2.27 \times 10^{-1}$ & $1.69 \times 10^{-1}$ & $1.27 \times 10^{-1}$ & $1.09 \times 10^{-1}$ \\
\hline
\end{tabular}

Table 4: $\left(\mathcal{W}_{h}^{1 / 4,1,0}\right)-\left\|T w_{T h}^{0}-\chi_{h}^{0}\right\|_{L^{2}(\Omega)}$ and $\left\|T w_{T h}^{1}-\chi_{h}^{1}\right\|_{L^{2}(\Omega)}$ vs. $T$ with $\varepsilon=10^{-8}, h=1 / 64$. 


\section{List of Figures}

$1 \quad\left(\mathcal{W}_{h}^{1 / 4,0,1}\right)$ - Initial condition $w_{h}^{0}$ (left) and $w_{h}^{1}$ (right) of the semidiscrete system $(22)$ on $\Omega=$

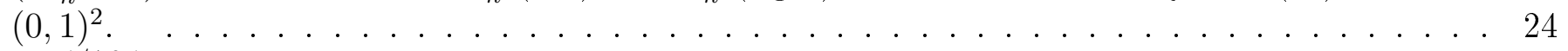

$2\left(\mathcal{W}_{h}^{1 / 4,0,1}\right)$ - Stabilization of initial condition defined by $(10)$ : Approximation $y_{h}(t)$ on $\Omega$ for (from left to right, top to bottom) $t=0,3 / 7,9 / 7,15 / 7,18 / 7$ and $T=3 \ldots \ldots 25$ 

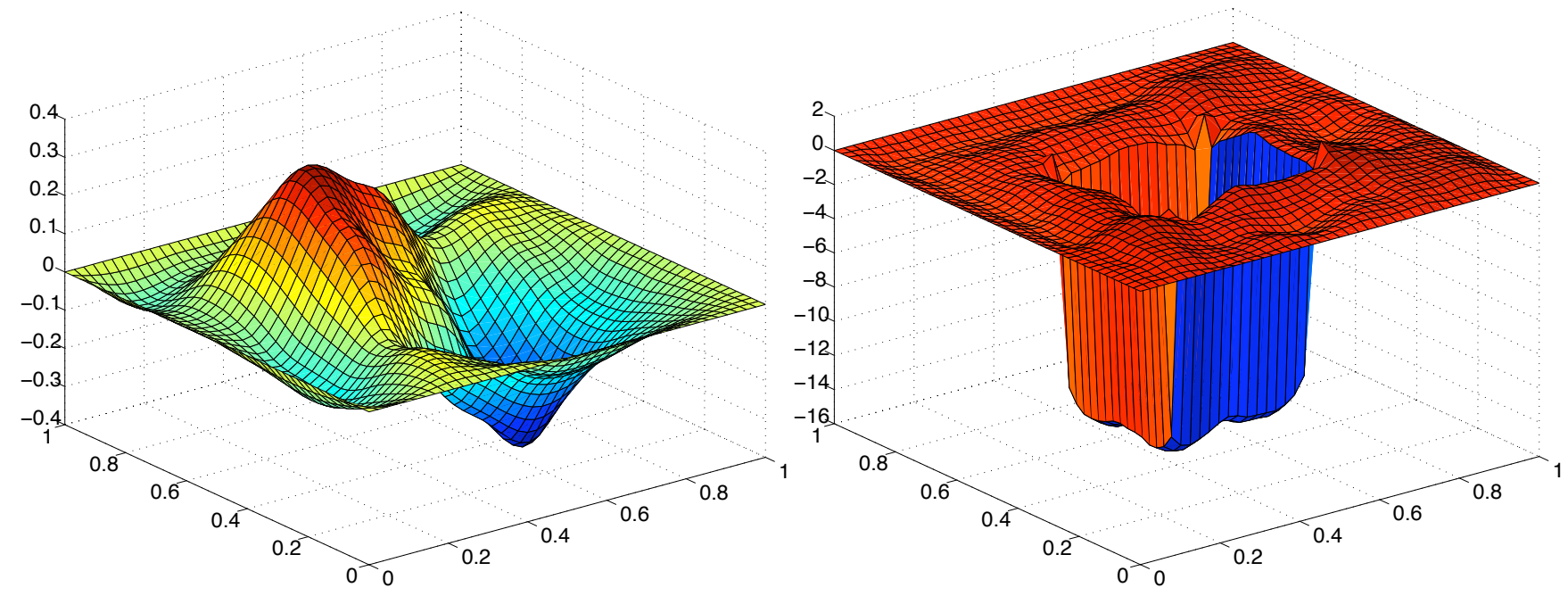

Figure 1: $\left(\mathcal{W}_{h}^{1 / 4,0,1}\right)$ - Initial condition $w_{h}^{0}$ (left) and $w_{h}^{1}$ (right) of the semidiscrete system $(22)$ on $\Omega=(0,1)^{2}$. 

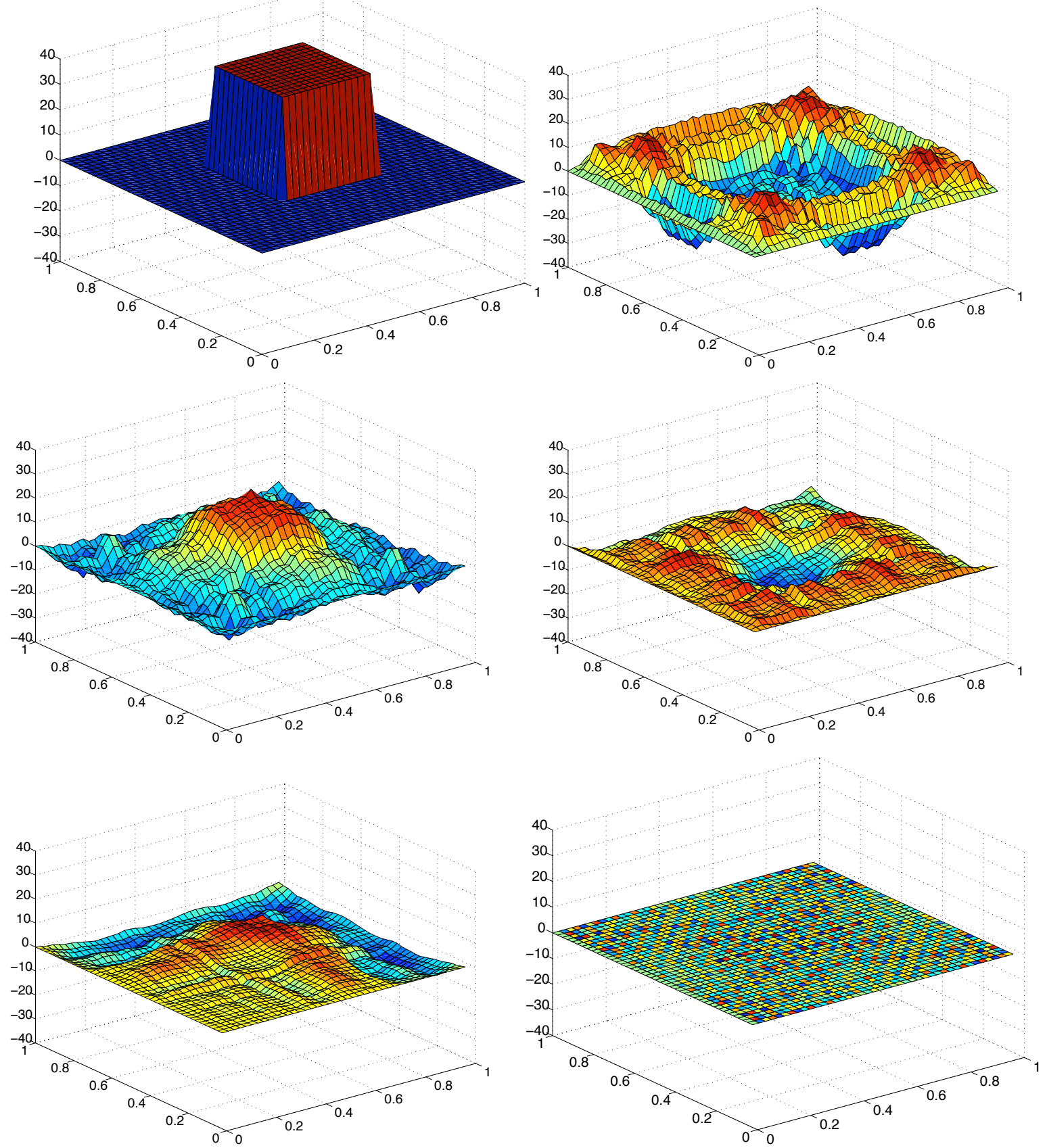

Figure 2: $\left(\mathcal{W}_{h}^{1 / 4,0,1}\right)$ - Stabilization of initial condition defined by (10): Approximation $y_{h}(t)$ on $\Omega$ for (from left to right, top to bottom) $t=0,3 / 7,9 / 7,15 / 7,18 / 7$ and $T=3$. 